\title{
Ion Flux Transmission Along an Insulating Duct with an Axial Magnetic Field
}

\author{
D. Grondona* H. Kelly*, and L. Giuliani \\ Instituto de Física del Plasma (CONICET), Departamento de Física, \\ Facultad de Ciencias Exactas y Naturales (UBA) \\ Ciudad Universitaria Pab. I, (1428) Buenos Aires, Argentina
}

Received on 30 December, 2003; revised version received on 28 April, 2004

\begin{abstract}
An experimental study of the metallic ion flux in a pulsed copper vacuum arc with an annular anode and operated with an axial magnetic field is presented. It is employed an insulating drift duct surrounded by an external coil which generates an axial magnetic field. Operating the arc under vacuum condition, measurements of the ion flux and the plasma potential at different axial positions along the duct and different magnetic field values are presented. The arc voltage and the total discharge current as functions of the magnetic field intensity are also reported. We find that the decay of the ion flux through the duct walls is not exponential. A simplified model for the ion emission is used in order to obtain an interpretation of the measured ion current for different axial positions and magnetic field strength, and a good agreement with the experimental points is found.
\end{abstract}

\section{Introduction}

The study of the ion transport through axial magnetic fields is of special interest for Vacuum Arc plasma sources. These devices are attractive for the deposition of thin films, since an intense jet of metallic plasma including relatively highenergy ions (15-120 eV, depending on the cathode material and charge-state of the ions) is produced with a high efficiency at the cathode surface[1]. The ions come from minute points on the cathode known as "cathode spots", and the ion current amounts to 8-10\% of the total discharge current [2]. However, cathodic arcs also produce macroparticles of the melted cathode material that are deposited on the substrate. The presence of macroparticles increases the porosity and roughness of the coating, affecting the films properties. Several filtering systems to separate the arc plasma and the macroparticles have been developed [3]. Presently, magnetic filtering is a widely used method for removing the macroparticles. This kind of filter consists of a tube with an axial magnetic field. The plasma flux is guided through the tube by the magnetic field, whereas the massive macroparticles [1], which are ejected mostly at large angles with respect to the cathode surface, bounce or stick on the duct walls. The tube can be a straight tube (rectilinear filter) $[4,5,6]$ or a curved one $[7,8,9]$. The main drawback of these filters is the ion losses along the filter, producing a significantly reduction in the deposition rate. For this reason, it is important to understand the mechanism of these losses, in order to optimize the filtering system.

In this work we study the plasma transport in a rectilinear filter with insulating walls. The plasma ion current and the floating potential of a collector located at different axial positions along the filter, and for different magnetic field intensities are measured. Also, the way the field affects the discharge parameters (arc voltage and arc current) is studied. The experimental data are compared with a simple conical model for the cathodic ion emission [6], and a good agreement with the measurements is found.

\section{Experimental setup}

A schematic of the experimental apparatus (PROTO 2CC) is shown in Fig. 1. The arc was pulsed for $35 \mathrm{~ms}$, with a peak current of $400 \mathrm{~A}$. It was produced by discharging an electrolytic capacitor bank with $\mathrm{C}=0.075 \mathrm{~F}$, connected to a series inductor-resistor $(\mathrm{L}=2 \mathrm{mH}, \mathrm{R}=0.33 \Omega$ ), which critically damped the discharge. A mechanically controlled tungsten trigger rod ignited the arc. The arc was operated in a high vacuum, with a background pressure of $0.01 \mathrm{~Pa}$.

A grounded copper cathode $(5 \mathrm{~cm}$ in length and $1 \mathrm{~cm}$ in diameter) was placed in front of an annular anode with an aperture of $5 \mathrm{~cm}$ and a length of $2 \mathrm{~cm}$. The cathode lateral surface was cover with a Pyrex insulator, so as to ensure that the ion emission was through the front surface of the cathode. The distance between the cathode surface and the closest plane of the anode was $1 \mathrm{~cm}$. At the end of the anode it was located the entrance of the magnetic duct. The magnetic field was established by three external coils wrapped around an insulating tube, $23 \mathrm{~cm}$ long and with an inner wall radius $r_{D}$ of $5 \mathrm{~cm}$. The coil was supplied with dc current from an independent power source. The magnetic field strength was measured with a calibrated Hall probe, and the magnetic field intensity $(B)$ was characterized with the value measured at the duct center. The maximum obtained magnetic field intensity was $253 \mathrm{G}$.

\footnotetext{
* Member of the CONICET
} 


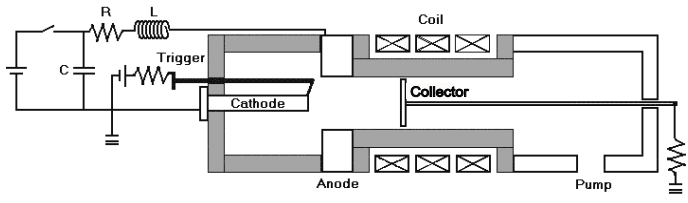

Figure 1. Scheme of the experimental apparatus (PROTO 2CC). Insulating pieces are indicated with gray color.

The arc produced a fully-ionized plasma jet with multiply charged plasma ions, in particular for copper the average charge is $z_{m}=1.85$ [10]. An aluminum collector plate was positioned perpendicular to the axis of the duct and could be moved axially along the duct. The collector plate radius $\left(r_{T}\right)$ was almost coincident with the duct inner radius, so the collector plate collected nearly all the charge axially flowing along the duct. The collector plate was floating to measure the floating potential $\left(V_{f l}\right)$ or it was connected to ground through a resistance to determine the ion current $\left(I_{i}\right)$ from the resistance voltage drop. The resistance value was selected enough low so as to ensure that the collector voltage was smaller than $V_{f l}$. Both $I_{i}$ and $V_{f l}$ were measured as functions of $B$ at several axial distances $(d)$ from the cathode surface.

The arc voltage drop $\left(V_{a c}\right)$ and the discharge current $\left(I_{d}\right)$ as functions of $B$ were measured using a resistive voltage divider and a calibrated small-value $\left(8 \times 10^{-3} \Omega\right)$ resistor, respectively.

\section{Results}

A typical profile of $B$ corresponding to a coil current of $26 \mathrm{~A}$ is presented in Fig. 2. It can be seen that the magnetic field strength remains almost constant along the tube, but it sharply drops at the duct extremes. For example, for a magnetic field of $130 \mathrm{G}$ in the middle of the duct, the magnetic field at the cathode region is about $30 \mathrm{G}$. For that reason, $B$ at the cathode region is too small to alter the discharge parameters.

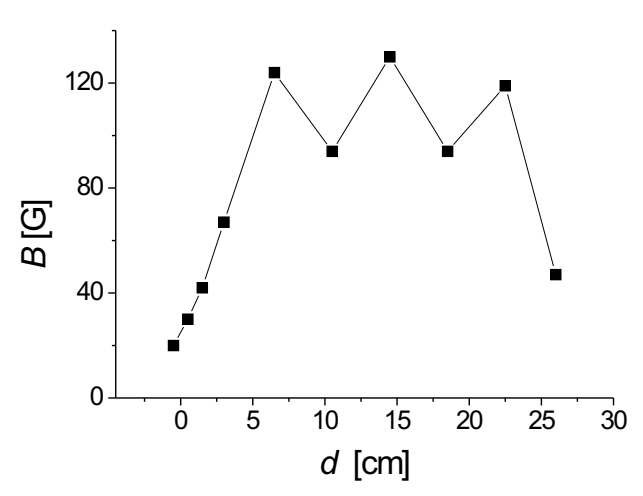

Figure 2. Typical profile of $B$ corresponding to a coil current of 26 A.

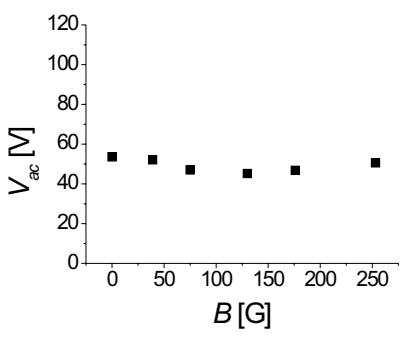

a)

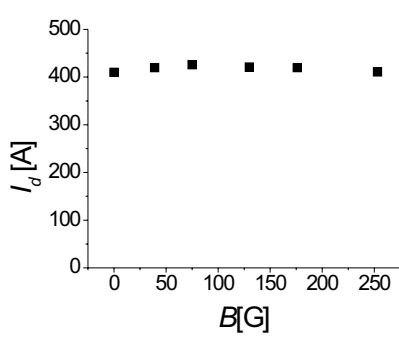

b)
Figure 3. Arc voltage vs. magnetic field intensity (a), and discharge current vs. magnetic field intensity (b).

In Fig. 3a) and 3b) $V_{a c}$ and $I_{d}$ vs $B$ are presented, respectively. Each point in the figures represents the average of five consecutive shots performed under identical conditions. It can be seen that both quantities are almost independent of $B$, and take the values $V_{a c} \approx 50 \mathrm{~V}$ and $I_{d} \approx 400 \mathrm{~A}$.

In some discharges without magnetic field, the annular anode was changed by a disk anode, and placed in front the cathode. In these discharges the measured value of $V_{a c}$ was approximately $10 \mathrm{~V}$ less than in the annular configuration ( $\approx 40 \mathrm{~V}$ ), and this fact indicated the presence of an anodic potential drop in the annular configuration.

In Fig. 4 the ion saturation current as a function of $d$ with $B$ as a parameter is presented, and in Fig. 5 the ion saturation current measured at the middle of the filter $(d=$ $14.5 \mathrm{~cm}$ ) as a function of $B$ is presented. It can be seen (Fig. 4) that $I_{i}$ decreases significantly with $d$ for $B$ values lower than $130 \mathrm{G}$, but for higher $B$ values, the ion current remains almost constant along the filter, with the only exception of the case $B=130 \mathrm{G}$, where the ion current decreases significantly at the filter exit. It can also be observed from both figures that $I_{i}$ increases with the magnetic field, and in particular, it shows an abrupt growth for $B$ values in the range of $50 \mathrm{G}$ to $130 \mathrm{G}$, and saturates for higher $B$ values.

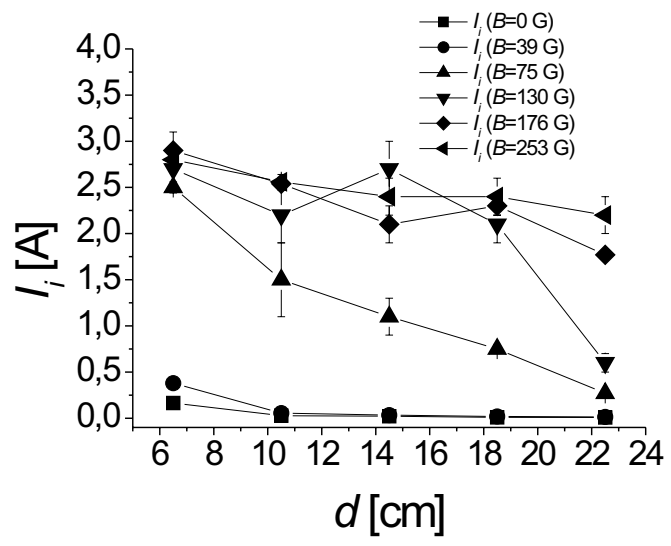

Figure 4. Ion saturation current as a function of $d$ with $B$ as a parameter. 


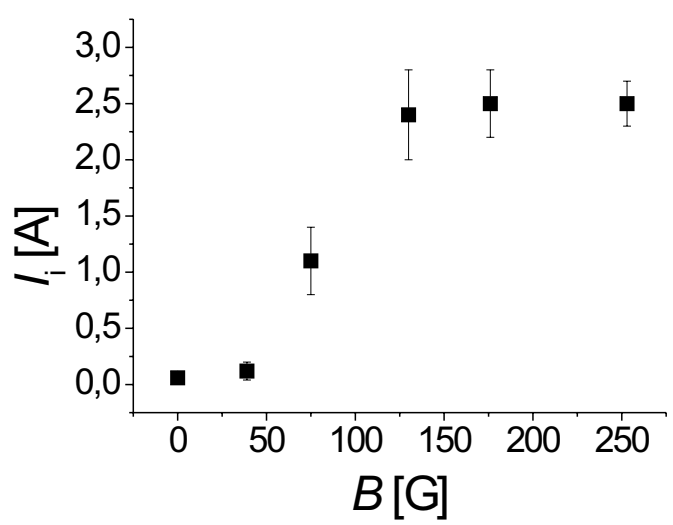

Figure 5. Ion saturation current measured at the middle of the filter $(d=14.5 \mathrm{~cm})$ as a function of $B$.

In Fig. 6 the collector floating potential vs $d$ with $B$ as a parameter is presented. It can be seen that $V_{f l}$ remains roughly constant along the filter and shows an increase with the magnetic field value. In Fig. 7 the collector floating potential measured at the middle of the filter as a function of $B$ is presented. It can be seen that $V_{f l}$ slightly increases with $B$ up to $B$ values of $130 \mathrm{G}$, and then remains almost constant $\left(V_{f l}=29 \pm 2 \mathrm{~V}\right)$ for higher $B$ values. This behavior could be related to slight variations of the plasma potential (of the order of the anode potential) with $B$, although it is difficult to establish any correlation between both potentials due to the experimental uncertainties.

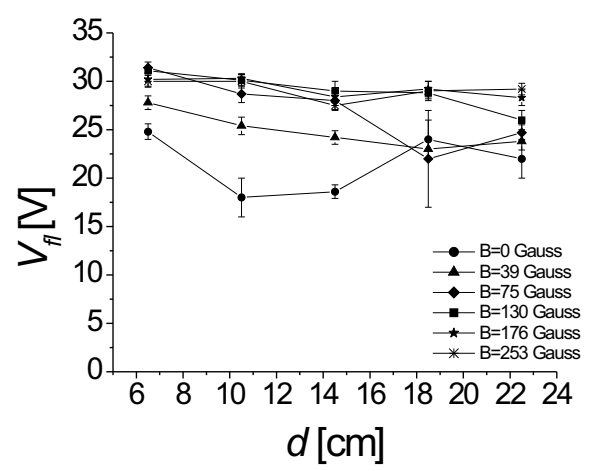

Figure 6. Collector floating potential vs $d$ with $B$ as a parameter.

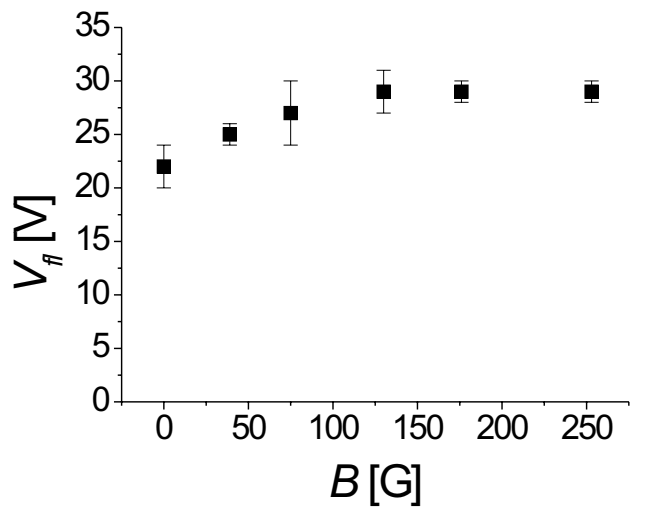

Figure 7. Collector floating potential measured at the middle of the filter as a function of $B$.

\section{Discussion and final remarks}

The first point to consider is an estimation of the plasma parameters in the duct in terms of the presented measurements. From the $I_{i}$ value for $B=0 \mathrm{G}\left(I_{i} \approx 0.2 \mathrm{~A}\right.$, see Fig. 4), assuming that the whole probe's area collects ions, and using Lam's electrostatic probe theory [11], the plasma density inside the duct can be estimated as $n \approx 3 \times 10^{10} \mathrm{~cm}^{-3}$. If it is assumed that probe's theory remains valid for the evaluation of the ion saturation current in the presence of $B$ perpendicular to the collecting probe surface, for $B=253 \mathrm{G}\left(I_{i}\right.$ const. $=2.5 \mathrm{~A}$, see Fig. 4), and assuming that the whole probe's area collects ions, the plasma density inside the duct can be estimated as $n \approx 3 \times 10^{11} \mathrm{~cm}^{-3}$. From the floating potential measurements for $B=0 \mathrm{G}$, and considering that the plasma potential $\left(V_{p l}\right)$ is given as the anode potential $(\approx 50 \mathrm{~V}) \mathrm{mi}$ nus the anodic sheath potential drop $(\approx 10 \mathrm{~V}),\left(V_{p l} \approx 40\right.$ $\mathrm{V})$, the electron temperature can be estimated as $\mathrm{T}_{e} \approx 4 \mathrm{eV}$ from the same theory [11].

In order to obtain an interpretation of the measured ion current for different $d$ and $B$ values, and following Ref [6], a simplified model for the ion emission is proposed. It is assumed that the ion emission with a total current $I_{o}$ and with an emission aperture angle $\alpha$ emerges from a virtual point $\mathrm{P}$ located at a certain distance $Z_{0}$ behind the cathode surface (of radius $r_{c}$ ). This geometry is illustrated in Fig. 8, where the gray zone indicates the massive cathode. The ion flow is assumed to propagate in a ballistic way along the duct, since the characteristic time for ion diffusion losses $\left(\tau_{\text {dif }} \sim 0.2\right.$ $r_{D}^{2} / \mathrm{D}_{a}$, where $\mathrm{D}_{a}$ is the ambipolar diffusion coefficient) results one order of magnitude larger than the ion transit time $t_{t r}$ along the duct $\left(\tau_{d i f} \sim 2 \times 10^{-4} \mathrm{~s} ; \tau_{t r} \sim 2 \times 10^{-5} \mathrm{~s}\right.$, for an ion velocity of $\sim 10^{6} \mathrm{~cm} / \mathrm{s}$ ).

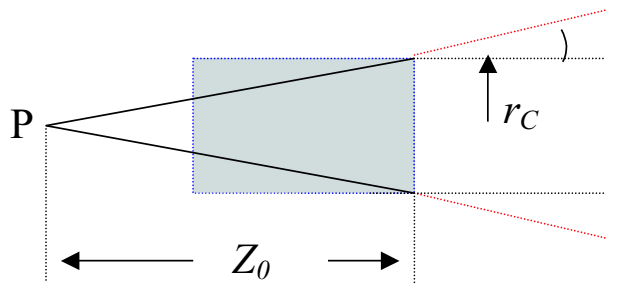

Figure 8. Scheme of the assumed model for the ion emission. The gray zone indicates the cathode.

The ion current $I_{i}$ collected by a circular plane surface of radius $r_{T}$ perpendicular to the symmetry axis and located at a distance $\mathrm{R}$ from $\mathrm{P}$, considering that the collector is completely covered by the ions, is:

$$
I_{i}=\left[I_{o} /(1-\cos \alpha)\right] \cdot\left[1-\left(1+r_{T}^{2} / R^{2}\right)^{-3 / 2}\right]
$$

where $\mathrm{R}=d+Z_{0}=d+r_{c} / \tan$ (see Fig. 8). For the case in which the ion flux is enough collimated so that the collector is only partially covered by the ions, $I_{i}$ is:

$$
I_{i}=\left[I_{o} /(1-\cos \alpha)\right] \cdot\left[1-\left(1+\tan ^{2} \alpha\right)^{-3 / 2}\right]
$$


In Fig. 9 the theoretical $I_{i}$ (calculated according to one or another of the above given equations) is plotted with lines as a function of $\mathrm{d}$ for $I_{0}=0.8 \mathrm{~A}$. The aperture angle was varied from $\alpha=0.1^{0}$ for $B=253 \mathrm{G}$ to $\alpha=85^{0}$ for $B=0 \mathrm{G}$.

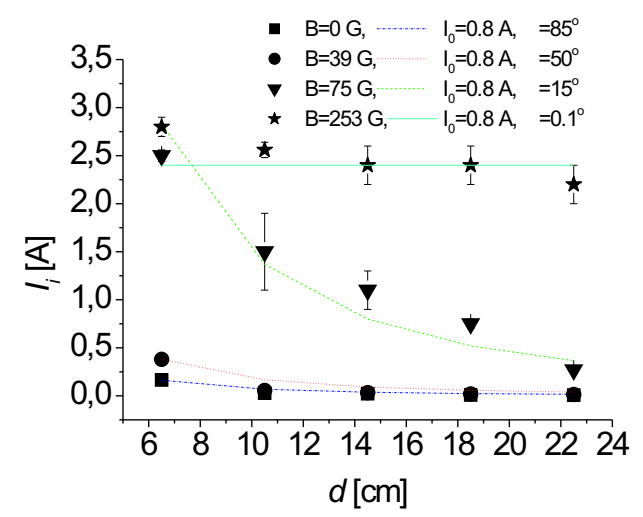

Figure 9. Theoretical (lines) and experimental ion current (scattered points) as functions of $d$ for $B=0,39,75$, and $253 \mathrm{G}$.

It can be seen from Fig. 9 that in all the cases there is a good fitting with the experimental values, indicating that the falloff of the ion current is not exponential (an exponential decay law should be expected if diffusion losses were dominant). Note that the value of $I_{0}$ predicted by the model is very low compared with the value of the total ion current emitted from the cathode $\left(\approx 0.1 I_{d} \approx 40 \mathrm{~A}\right)$. Hence an important fraction of the ions are lost in the cathode-anode region, where the main discharge develops. After emerging from the anode aperture and entering into the filter, the attenuation of the ion current is due to duct-wall losses, corresponding to a ballistic ion flux hitting the filter walls. These duct-wall losses are smaller for large $B$ values because in these cases the ion flux is more collimated ( decreases with $B)$.

With the knowledge of $\mathrm{T}_{e}$, the electron and the ion gyroradius can be evaluated as functions of $B$. The electron gyroradious is of the order of the duct radius for a very low magnetic field value $(B \approx 2 \mathrm{G})$, so the electrons are confined along the magnetic lines for all the range of $B$ values studied in this experiment. The ion gyroradious is of the order of the duct radius for $B=95 \mathrm{G}$ (the ion temperature has been taken to be of order $0.3 \mathrm{eV}$ [12], so for lower magnetic field values, only the electrons are confined, and some authors claimed $[4,5]$ that in this conditions ions are lost to the walls and hence the plasma is charged negatively. This charge induces radial electric fields in the plasma that reduce the rate of ion loses. For magnetic field values larger than $95 \mathrm{G}$, the ions are also partially confined, and a strongly decreased plasma loss rate should be expected because of the collimating effect of $B$ on both kinds of particles. Therefore, a saturation ion current must be expected at $B \approx 95 \mathrm{G}$, in good agreement with our experimental results.

\section{Acknowledgements}

This work was supported by grants from the Universidad de Buenos Aires (PID X214) and from the Agencia Nacional de Promoción Científica y Tecnológica (PICT 03-09491).

\section{References}

[1] R. L. Boxman, D. M. Sanders, and P. J. Martin; Handbook of Vacuum Arc Science and Technology, Fundamentals and Applications, Park Rige, NJ: Noyes, 1995

[2] C. W. Kimblin, J. Appl. Phys. 44, 3074 (1973).

[3] M. Krzysztof, J. Walkowicz, and J. Bujak, Plasma \& Ions 3, $41(2000)$

[4] J. Storer, J. E. Galvin, and I. G. Brown, J. Appl. Phys.66, 5245 (1989).

[5] B. P. Cluggish, IEEE Trans. Plasma Sci. 26, 1645 (1998).

[6] H. Kelly, L. Giuliani, and F. Rausch, J. Phys. D: Appl. Phys. 36, 1980 (2003).

[7] I. I. Aksenov, V. A. Belous, V. G. Padalka, and V. M. Khoroshikh, Sov. J. Plasma Phys. 4, 425 (1978).

[8] R. L. Boxman, V. N. Zhitomirsky, B. Alterkop, E. Gidalevich, M. Keidar, and S. Goldsmith, Surf. Coat. Technol. 86-87, 243 (1996).

[9] S. Anders, A. Anders, and I. Brown, J. Appl. Phys. 74, 4239 (1993).

[10] J. Kutzner and H. C. Miller, J. Phys. D: Appl. Phys. 25, 686 (1992).

[11] S. H. Lam, Phys. Fluids 8, 73 (1965).

[12] F. Minotti, H. Kelly, and A. Lepone, Plasma Sources, Sci. and Technol. 11, 294 (2002). 